\title{
Los 10 frentes de la comunicación corporativa
}

\section{The Ten Fronts of Institutional Communication}

José Carlos Losada Díaz. Universidad Católica San Antonio de Murcia

Recibido 11-XI-2008 - Aceptado 23-III-2009

Resumen:

Es muy complicado que una disciplina tan vinculada a la labor profesional como la comunicación corporativa no sea capaz de encontrar un equilibrio entre su constante renovación impulsada por las cambiantes necesidades de la economía de mercado y un acuerdo de mínimos sobre cuál debe ser el núcleo de trabajo real de esta disciplina. El presente artículo trata de ofrecer los que consideramos que son los diez frentes que deben orientar la labor profesional del gestor de la comunicación corporativa en los próximos años.

Palabras clave:

Comunicación corporativa, comunicación de crisis, RSC, imagen, marca, identidad corporativa

Abstract:

Corporate Communication, as a field of knowledge linked to the professional work, finds hardly the balance between its renovation, stimulated by the needs of the market economy, and the agreement of the academic core of this field. The aim of this paper is to offer ten key points that should orientate the corporate communication manager work in the future.

Key Words:

Corporate Communication, Crisis Communication, RSC, Image, Brand, Corporate Identity 


\section{Introducción}

La comunicación corporativa ha experimentado un crecimiento significativo en nuestro país en los últimos años. Ha dejado de moverse en el terreno de la reivindicación para posarse en la realidad empresarial de forma firme y continuada, ocupando un espacio que no existía o que ha contribuido a dar un valor que no tenía. Como asegura López Triana, la comunicación ha dado un paso enorme descubriendo "nichos de valor que antes o se desconocían o se despreciaban, y al hacerlo, se ha convertido en generadora de valor, de diferenciación y de liderazgo" (Villafañe, 2008:13). De este modo, la comunicación se ha posicionado como un factor estratégico indudable, muy lejos de los comienzos titubeantes en los que suponía un mero adorno estético en el engranaje empresarial o un factor puramente comercial. Ha costado muchos años para que en nuestro país la comunicación se haya alejado suficientemente de este territorio y haya alcanzado un peso sustancial en la realidad empresarial.

Podemos observar el crecimiento de la comunicación corporativa tanto desde una perspectiva cualitativa como desde una cuantitativa. Empecemos por este segundo punto, porque los datos, en este sentido, son muy claros. Según un amplio estudio ${ }^{1}$ realizado desde Adecec (Asociación de Empresas Consultoras en Relaciones Públicas y Comunicación), ocho de cada 10 empresas consultadas tienen servicios propios de comunicación (en concreto, el 82,2\%) y la mitad de ellas contrata a una o varias agencias especializadas en áreas concretas. Además, el mismo estudio confirma que el 91,2\% de las empresas afirman realizar labores de "comunicación corporativa" y casi la mitad, el 49\%, consideraban que la función de comunicación en la empresa y, en general, el sector, mantendrían el crecimiento durante los próximos años. Como aportación significativa, el mismo estudio recordaba que, en la actualidad, el gasto medio en labores de comunicación supera los 361.000 euros al año.

En lo meramente cualitativo, el avance también ha sido evidente. Así, hay una larga distancia entre lo que empezaron siendo los servicios de comunicación hace 20 ó 25 años y lo que ahora son sus responsabilidades. De este modo, la mayoría de las prácticas profesionales de cierto calado que arrancaron con la llegada de la democracia en los años 70 se asociaron casi en exclusividad a las relaciones con los medios de comunicación, que también sufrieron un importante desarrollo durante estos años. Era una forma de entender la comunicación en las organizaciones desde una perspectiva puramente periodística. Es decir, las organizaciones entendían que la única necesidad desde la cual construir la comunicación externa era mejorar las relaciones con los medios de comunicación. Por ello, la práctica profesional se reducía a elementos propios de los medios de comunicación y los profesionales demandados no dejaban de ser periodistas reconverti-

\footnotetext{
1 http://www.adecec.com, según estudio de Sigma Dos, abril de 2008, encuestando a las 516 empresas más importantes del país.
} 
dos. Junto con esta tendencia, existía una complementaria, como era la de entender la comunicación como una parcela más del marketing de la empresa, es decir, de su política comercial.

No ha sido sencillo deslizarse fuera de estos dos campos de juego -que, por supuesto, siguen siendo importantes- pero la llegada de nuevos profesionales, la importación de técnicas y metodologías y el desarrollo de la vertiente académica han logrado que los gestores de comunicación se adentren en el terreno de los intangibles, ese conjunto de variables que añaden o incluso modifican el valor de las organizaciones a las que se asocian y que, por lo tanto, forman parte directa de su actividad diaria. Hablamos de cuestiones como la Responsabilidad Social Corporativa, gestión del conocimiento, reputación corporativa, gestión de marca y otros campos de actividad en las que la comunicación -o gracias a ella- ha ido encajando en los últimos años en la misma medida que su peso en la empresa.

Existe una incuestionable percepción generalizada de que la comunicación corporativa -normalmente materializada en los directores de comunicación o dircom- es una parte indiscutible de la dirección de cualquier organización. José Manuel Velasco, dircom de Unión Fenosa y presidente de la asociación Dircom, lo justifica asegurando que la comunicación "es un catalizador, el elemento que provoca las reacciones adecuadas" 2 . Un hecho lo demuestra: la comunicación corporativa ha ido ocupando una parte importante dentro del staff de las más grandes compañías, dependiendo casi en exclusiva de los órganos de Dirección de la compañía, ya sea la Presidencia, el Consejo de Administración u otro órgano. Hay muchos ejemplos: en Enagás, la comunicación es una de las ocho direcciones generales que dependen directa y exclusivamente de la Presidencia de la Compañía; en Abengoa, la comunicación es una de la docena de áreas corporativas de las que dependen todas las unidades de negocio; en Endesa, su Dirección de Comunicación es una de las siete direcciones generales de la organización por debajo, solamente, del Presidente Ejecutivo y del Consejero Delegado; o la ya mencionada Unión Fenosa, cuyo Gabinete de Comunicación se sitúa en el organigrama justo por debajo del Presidente. Los ejemplos son incontables.

No sólo eso. El crecimiento en lo profesional ha sido paralelo a lo académico. Dos datos ilustran esta afirmación. El primero es un hecho académicamente muy relevante: la inclusión de la comunicación como una de las materias básicas dentro de la rama de conocimiento de las Ciencias Sociales y Jurídicas en el nuevo ordenamiento de las enseñanzas universitarias ${ }^{3}$, lo que implica un peso sustancialmente mucho mayor con respecto a otros ámbitos del saber. Nunca había ocurrido antes y, de hecho, hace pocos años era algo absolutamente impensable.

En “Más responsabilidad para el dircom”, en El País, 23 de noviembre 2003 (Suplemento Negocios, 37)

Real Decreto 1393/2007 de 29 de octubre. 
El otro dato es el crecimiento exponencial de facultades universitarias en las que se han implantado estudios de comunicación en España (casi setenta en la actualidad) y el número de títulos de postgrado (Doctorado y Master) que se ofertan en este ámbito en centros universitarios y escuelas de negocios, que superan ampliamente los 200. En el terreno de los postgrados, un porcentaje mayoritario están dedicados a la comunicación corporativa. Son datos que evidencian un crecimiento innegable del valor académico y de la formación en este sector.

Pero esta evolución cuantitativa y cualitativa a la que nos estamos refiriendo tiene consecuencias, también, negativas. Y, probablemente, la más importante de ellas sea el desorden conceptual y profesional que parece que se ha instalado como algo incluso consustancial al propio desarrollo de la disciplina. Cada vez parece más difícil distinguir cuáles son las actividades propias de la comunicación corporativa, con lo que no hace que sea sencillo tener claros sus cimientos y sus parcelas de desarrollo en los próximos años. Es aquí donde encaja plenamente el objetivo de este texto: en tratar de lanzar abiertamente los que consideramos que son los diez frentes de acción del responsable de comunicación corporativa en los próximos años, sus campos prioritarios de desarrollo profesional y académico.

Este es el objetivo principal del presente artículo, la localización de los diez principales ejes de actuación que constituyen las responsabilidades inherentes al cargo de un gestor de la comunicación corporativa a día de hoy, las claves sobre las que orientar su labor, teniendo en cuenta el actual contexto empresarial y económico sin el cual la aportación de la comunicación hubiera sido muy diferente al que es: el desarrollo de la economía de servicios, la globalización de empresas y marcas y el incremento exponencial del valor de los intangibles en estos últimos años.

En todo caso, las particularidades de cada sector y sus necesidades específicas implicarán otras funciones diferentes o simplemente más específicas que tengan que ver con su negocio o con sus públicos. No obstante, consideramos que los siguientes son una representación lo suficientemente completa como para asegurar que, difícilmente, pueda existir algo que quede al margen. Este conjunto de ejes principales de actuación de la comunicación corporativa serían los que siguen a continuación:

\section{Relaciones con los medios de comunicación}

El conjunto de relaciones con los medios de comunicación es, probablemente, la dimensión que más preocupa a las compañías actuales y a sus directivos. Es la clave principal de la comunicación corporativa desde hace años. De hecho, en muchos casos, llega a convertirse en una obsesión por todo lo que significa "salir 
en los medios", una "ansiedad mediática" que difícilmente se ajusta a la realidad de la compañía o, incluso, a la política informativa de los medios. Más allá de esto, lo cierto es que estas relaciones entre empresas y medios persiguen un doble objetivo: para las compañías, potenciar la proyección positiva en los medios y, para los medios, proporcionar información actual de calidad sobre lo que ocurre en su ámbito de competencia informativa. Estos intereses mutuos son en la gran mayoría de los casos conjugables, siempre que se basen en un tipo de relación permanente y profesional basada en los principios tan irrenunciables como el servicio y la credibilidad. Álvarez y Caballero (1997: 211) llegan a asegurar que "el contacto institución-periodista es fruto de un interés y obligación mutua. La primera debe suministrar, explicar y contextualizar las informaciones. El periodista se dedica a recoger y verificar en sus distintas fuentes de información. Tener conciencia de esta dependencia mutua beneficia a ambas partes”.

No es fácil conciliar, en muchos casos, las necesidades de información de los medios y la proyección positiva que buscan las empresas. En muchas ocasiones, no buscan lo mismo. Pero, cada vez con más fuerza, el modo de trabajar de ambas partes se acerca a uno de los escasos puntos de conexión seguros entre los intereses mutuos: la empresa como fuente confiable para el periodista. No es una declaración sin más. Supone un modo de funcionar, una prioridad y hasta un claro posicionamiento, incluso en cuestiones de carácter ético. Olga Martínez (2007) lo define así: "Se trata de trabajar en equipo. Ellos necesitan publicar la información y nosotros aparecer en los medios. En lugar de temerlos tanto colaboremos con ellos y facilitemos su trabajo".

Las relaciones informativas tienen muchas posibilidades para la compañía. Pero eso no quiere decir que deba convertirse en una prioridad total o que las enfoquemos desde un modo tradicional y hasta obsoleto. Quizás, no se están aprovechando todas las opciones que ofrece la realidad profesional, ya que existen todavía ciertas tendencias muy consolidadas que cuestan superar a los nuevos comunicadores institucionales. De este modo, por ejemplo, no se segmentan los medios de comunicación, no se facilitan encuentros con los profesionales ni se opta por fórmulas creativas para la "venta” de nuestros mensajes. Pero esto es algo que, sin duda, irá cambiando, puesto que la propia dispersión y multiplicación de los medios de masas, de los gabinetes, y la globalidad de la comunicación -y , por lo tanto, del interés informativo- acabarán contribuyendo al incremento y a la complejidad de las relaciones y, por ello, también, al de las responsabilidades concretas que se le pueden exigir al responsable de comunicación y, entre las que están las siguientes:

- $\quad$ Construcción de mensajes informativos y de actualidad

- Elección y confección de los instrumentos necesarios para transmitir los mensajes en cada caso 
- $\quad$ Organización de "puestas en escena": eventos, ruedas de prensa...

- $\quad$ Elección de los medios a los que hacer llegar los mensajes (selección, adecuación, segmentación)

- $\quad$ Contactos individuales con los periodistas

- $\quad$ Seguimiento de medios y análisis de contenidos, así como su gestión y archivo documental

- Identificación de focos de posible interés por tipo de medio, por sectores y profesionales

Todas éstas son las responsabilidades mínimas que determinan la actividad de un responsable de comunicación en relación con los medios de comunicación, el tradicional gabinete de prensa, que utiliza los instrumentos tradicionalmente válidos para hacer llegar sus mensajes a los medios: Contacto directo, Nota de Prensa, Comunicado Oficial, Rueda de Prensa y Entrevista. Estos siguen siendo los medios habituales de contacto.

Si lo analizamos críticamente, no es posible observar cambios sustanciales en la práctica de las relaciones informativas en los últimos años, a pesar de la importancia que siempre se le ha concedido, o precisamente por ello. Al margen de cuestiones minoritarias, el único avance significativo puede haber consistido en la profesionalización de los servicios para periodistas dentro de las webs corporativas, un fenómeno que podemos observar en todas las empresas de peso en nuestro país, como ACS, Banesto, Telefónica, Gas Natural, Unión FENOSA, Iberia o RENFE. Es la denominada Sala de Prensa, a través de la que, cada vez más, se canalizan las relaciones entre medios de comunicación y la empresa. La estructura de estos lugares virtuales es muy similar en todos los casos, y viene determinada por la existencia de elementos como la fototeca, notas de prensa y comunicados oficiales, identidad visual, entrevistas, documentos audiovisuales, documentos especiales -boletín interno, informe anual, estadísticas, presentaciones-, enlaces de interés, dossieres, discursos institucionales y, por supuesto, los datos de contacto del departamento de comunicación. Algunas de estas salas de prensa incluyen un acceso restringido a determinadas áreas de la web para todos aquellos periodistas y medios de comunicación que se hayan registrado. Sea como sea, lo cierto es que existe una tendencia muy clara a institucionalizar la Sala de Prensa como el canal prioritario para el conjunto de relaciones con los periodistas.

Un apunte más. Por mucho que la realidad entre medios y empresas no haya variado sustancialmente estos años no implica que las relaciones mutuas sean las mejores. Simplemente, la rutina productiva ha ganado peso sobre otras cuestiones estratégicas o, simplemente, creativas. Esto provoca un desencuentro, nada infrecuente, entre lo que quieren los medios y lo que le ofrecen las empresas. Así, según el informe Periodistas, Empresas e Instituciones ${ }^{4}$, cuando hace referencia a las propuestas informativas -notas de prensa- enviadas

4 Elaborado en 2006 por Estudio de Comunicación y Demométrica, con el apoyo de la Federación de Asociaciones de Periodistas de Es- 
por las empresas a los medios de comunicación, se concluye que, en su mayoría, "no están redactadas con mentalidad periodística, son muy publicitarias, no incluyen suficiente información, tienen mucha "paja” y son largas, repetitivas y generalistas”. Una descripción aterradora. Esto puede explicar que sólo el 12\% de las propuestas informativas de las empresas son publicables -que no llegan al 8,6\% en la televisión- o que una de cada tres convocatorias hacia los medios son irrelevantes, porcentaje que alcanza el 45,8\% cuando hablamos de televisión. Deberíamos esforzarnos en no perder la perspectiva de lo que hacemos y para quién lo hacemos.

\section{Comunicación de crisis}

Una de las funciones con un peso específico cada vez más importante es la gestión comunicativa de las crisis. Los gestores saben que su organización puede verse sumida en una crisis de mayor o menor calado en cualquier momento y que, en ese instante, una reacción rápida, coherente y planificada en materia de comunicación puede minimizar el impacto producido por los acontecimientos no deseados e, incluso, en ocasiones, puede convertirse en un modo de fortalecer la imagen de la organización.

El objetivo de toda gestión comunicativa de una crisis es revertir el daño y convertirlo en una oportunidad para presentar a la compañía de otro modo. Es decir, la posibilidad de mostrar a nuestra empresa como una compañía capaz de responder de forma rápida y eficaz a los problemas que surgen en su actividad normal, mostrarla como una empresa que se presenta como una organización responsable y comprometida, aprovechar la crisis para encontrar nuevos líderes internos en la organización o para movilizar al conjunto del personal o, simplemente, aprovechar la crisis para que la empresa pueda aprender la lección, adopte enfoques nuevos, y realice análisis innovadores sobre el sentido de su actividad y de su organización. Todas éstas son oportunidades que la empresa puede aprovechar interna y externamente para su propio fortalecimiento, sin olvidar que las crisis, en no pocas ocasiones, son un problema de percepción, de imagen, y en este terreno la comunicación tiene un papel fundamental.

La fase más decisiva para la correcta gestión de una crisis es, curiosamente, antes de que ésta estalle. Esto quiere decir que buena parte del éxito de la gestión de una situación de crisis se decide antes incluso de que se aprecien indicios de su llegada. Es esta la fase en la que la organización se prepara con todos los elementos e instrumentos a su alcance. Es el momento de detectar los peligros y oportunidades y de establecer el protocolo de actuación para cuando llegue el momento de ponerlo en marcha. Es el momento para el aná-

paña (FAPE), la Asociación Nacional de Informadores de la Salud (ANIS) y la Asociación de Periodistas de Información Económica (APIE), y disponible en http://institucional.apmadrid.es/ACM_Upload/276YAI3142006.pdf 
lisis de las crisis potenciales a las que la empresa está expuesta, para la constitución del Comité de Crisis órgano que centraliza la gestión de las crisis-, para la preparación y entrenamiento del personal -simulacros de crisis, media-training- y, sobre todo, para la redacción del Manual de Crisis, documento esencial en el se establecen las pautas fundamentales, el protocolo de actuación, que la empresa debe seguir en el momento en el que estalle la crisis.

Existen muchos modelos para la gestión comunicativa de la crisis, pero para los objetivos de esta breve exposición, es útil referirse brevemente al modelo de las "Cuatro Rs" de Heath (1988), que incluye las siguientes etapas: Reducción, Preparación, Respuesta, Recuperación (Reduction, Readiness, Response, Recovery). En estas cuatro fases se concretan las responsabilidades siguientes: evaluación de los riegos y su posible impacto, el entrenamiento, el análisis del impacto de la crisis y activación de los mecanismos de recuperación. Cuatro fases, cuatro períodos, que centran el conjunto de responsabilidades de gestión de estas situaciones cada vez más comunes y a las que las organizaciones deben responder de modo profesional, valorando la importancia que la comunicación puede tener en su resolución exitosa.

Si nos asomamos a la realidad profesional, y según los datos de Dircom ${ }^{5}$ en referencia a las grandes empresas españolas, el panorama no es muy alentador. Sólo el 8,3\% de estas empresas cuentan con un presupuesto específico para la gestión de crisis, aunque el 29,2\% de las empresas realizan en alguna ocasión simulacros de crisis y el 43,1\% asegura haber llevado a cabo un simulacro de crisis en los últimos años.

\section{Portavocía}

Ser portavoz significa, básicamente, ser el rostro visible de la organización, la persona que encarna el mensaje institucional, la fuente oficial. Los portavoces son figuras muy comunes en el mapa empresarial actual $^{6}$, puesto que son ellos los que encabezan o personifican las relaciones de la empresa con el mundo exterior, en especial con los medios de comunicación, un tipo de relación que necesita de la unificación de la voz institucional para comodidad de los medios y para confianza de la organización (especialmente en situaciones de crisis, donde la figura del portavoz es imprescindible). Normalmente, las funciones de portavocía son ocupadas, como es natural, por el propio director de comunicación -la máxima autoridadpero no es difícil encontrar organizaciones en las que esta función es ocupada por una persona designada específicamente, o simplemente, por un alto directivo de la organización.

\footnotetext{
www.dircom.org

También en la Administración Pública, donde alcanzan la categoría de ministros o consejeros.
} 
Las funciones del portavoz son amplias y diversas, y suponen la más real personificación de la organización hacia el exterior. Pizzolante (2006: 158) se refiere al portavoz como "aquel que debe dar una entrevista a la prensa o el que asiste a un evento al cual es invitada la empresa, pensando y con razón que, al ser formalmente una persona entrenada, ésta asegura la correcta comunicación de sus mensajes”. En esencia, el portavoz debe ser capaz de transmitir mensajes formales e informales de su organización ante diversas audiencias, sean empleados, comunidades, vecinos, amigos, representantes sindicales, gremiales o políticos, congresos, asambleas, administración pública o medios de comunicación. Y para hacerlo de forma eficiente, como asegura Pizzolante (2006:159), "necesita acercarse a su público con un actitud adecuada, un interés sincero por compartir algo que considera bueno y valioso, consciente de quién es la audiencia y cuáles son sus intereses". No es una labor sencilla ni intrascendente. Así, a la figura del portavoz le acompaña una paradoja, como asegura Canel (2007: 214), cuando afirma que “(...) mientras que su poder se "reduce" a portar la voz de la institución (...), en la práctica, llevar a cabo esta función tiene gran relevancia, pues la consecuencias en la opinión pública pueden modificar la marcha de la organización”.

Pero la portavocía no acaba en la figura del portavoz. La Portavocía también debe incluir el "servicio interno" que recoge la formación y entrenamiento de las habilidades de comunicación de todas aquellas personas que, en la organización, puedan necesitar ejercer como líderes internos o externos en determinados contextos. Es, por lo tanto, una visión más amplia de la portavocía, la del entrenamiento interno al personal que pueda utilizar sus habilidades en contextos como los siguientes que apunta Maribel Reyes (2007):

- $\quad$ Presentaciones corporativas

- $\quad$ Relaciones Informativas

- $\quad$ Encuentros con directivos

- $\quad$ Reuniones Internas

Como vemos, todas ellas son situaciones más o menos comunes en la organización moderna, situaciones cuyo denominador común es que la información se transmite personalmente, sin interlocutores, con lo que el centro del mensaje se centra en las personas y no tanto en la perfección del contenido. Las personas son las protagonistas y ellas son la encarnación de la empresa frente a los públicos con los que se encuentra?

De ahí arranca la importancia que destaca Reyes (2007: 35): “Son numerosos los casos que ponen en evidencia la necesidad de tener un representante principal conocido por los diferentes públicos (...) tanto en situaciones de comunicación corporativa, orientadas a reforzar la imagen de marca, como de comunicación interna e intermedia, en la que los destinatarios son los accionistas, directivos, 


\section{Diseño y realización de eventos}

El diseño y realización de eventos es un área de creciente interés para las compañías, que están descubriendo cómo este tipo de actividades pueden funcionar como vehículos enormemente potentes ${ }^{8}$ para la transmisión de determinados mensajes a sus audiencias. Por eso, desde un punto de vista estratégico, se han convertido en un verdadero instrumento de comunicación, en oportunidades para obtener un tipo de visibilidad al margen de los medios convencionales. Como afirma Canel (2007: 265), "los eventos no suceden, no son acontecimientos espontáneos. Son, más bien, sucesos que se idean con el objetivo de contar algo". Esa es precisamente la función nuclear de los eventos: contar algo, hacer llegar un mensaje a través de acontecimientos singulares capaces de aportar información de valor para ciertos públicos, sobre todo, para los externos. Por eso, deben ser responsabilidad directa del director de comunicación externa.

Cuando hablamos de eventos nos estamos refiriendo a una amplia gama de acontecimientos públicos ${ }^{9} \operatorname{con}$ los que la compañía busca la creación de determinadas experiencias directas en contacto con alguno de sus públicos prioritarios (empresarios, accionistas, administración pública, ciudadanos...) tratando con ello de hacerles llegar algún mensaje. Esta experiencia directa es un enorme canalizador de imagen para la empresa, muchas veces mayor que gran parte de los instrumentos de comunicación más habituales, ya que se elimina por completo cualquier tipo de mediación con el público final que, de esa forma, puede "convivir" con la marca de forma directa. Si esta experiencia es positiva y significativa, de forma directa o indirecta, consciente o no, se acaba traduciendo en notoriedad o en imagen para este público.

Los eventos no dejan de ser una oportunidad de visibilidad de la empresa y una opción de enfocarla, además, en determinada dirección. Pero no debemos olvidar algunas condiciones decisivas en la creación y gestión de estos eventos. En primer lugar, que los eventos deben partir de una necesidad concreta y, por lo tanto, de unos objetivos de comunicación delimitados por la compañía, lo que obliga a una investigación previa, sin la cual el diseño del acontecimiento tendrá una consideración estratégica. Será, simplemente, una presentación espectacular, pero nada más. En segundo lugar, si se hace de un modo adecuado, el evento

mandos intermedios, empleados y colaboradores, e incluso de situaciones de marketing, cuando se impone apoyar la imagen de los productos y servicios".

8 Su importancia creciente se demuestra si hacemos caso al informe "Retos, Tendencias y Oportunidades en el sector de eventos", publicado en 2007 a través Terra Consultoría de Incentivos y Mind Value y dirigido por Francisco Alcalde, estudio que confirma que el sector de eventos alcanza ya el 15\% del presupuesto destinado por las empresas españolas al marketing, aunque de momento esta cifra sólo sea la mitad de la que se destina a estos fines en el Reino Unido.

9 Actos que pueden ser de todo tipo, desde seminarios a fiestas, pasando por inauguraciones, conferencias, presentaciones, actos deportivos, actos culturales y un largo etcétera, en el que entra la capacidad de la organización de ser, a la vez, estratégico y creativo, es decir, crear el evento adecuado desde un punto de vista empresarial y comunicativo. 
debe proporcionar a la compañía resultados que se puedan medir, es decir, que sea posible comprobar realmente el efecto que ha tenido sobre la notoriedad o cualidades asociadas a la marca de la compañía en el público objetivo ${ }^{10}$. Todos los acontecimientos creados sin considerar estas dos necesidades se quedarán en una fórmula meramente estética, sin rendimiento para la empresa y con un éxito difícil de prever.

\section{Estudios de Imagen, de Notoriedad y de Comunicación}

Una de las funciones más decisivas en la moderna gestión de la comunicación es, sin duda, la de "vigilar" el conjunto de relaciones que mantiene la organización con sus públicos y, por supuesto, el resultado de dichas relaciones, es decir el estudio permanente del funcionamiento de la comunicación y del conjunto de percepciones manejadas por sus públicos. Nos estamos refiriendo al análisis regular de cómo funcionan los resortes de comunicación que estamos utilizando y cuál es la imagen que nuestros públicos tienen de nosotros como resultado de ello:

\subsection{Estudios de Imagen y de Notoriedad}

Uno de los objetivos generales que hemos definido para los órganos de comunicación externa es cuidar y mejorar la imagen que de la organización tienen sus públicos externos, que no es otra cosa que la percepción que éstos tienen sobre la compañía o sus productos. La imagen ${ }^{11}$ es un fenómeno complejo, sin duda, y su formación responde a una lógica que al comunicador le toca entender para tratar de orientarla hacia el beneficio institucional, algo que no siempre es posible, puesto que la responsabilidad última de la imagen es siempre de los públicos externos y éstos no siempre se comportan de modo previsible. Por ello, es tan necesario ir pulsando la vitalidad y la fortaleza de nuestra imagen, para tratar de ir reconduciendo las posibles desviaciones negativas que se puedan ir produciendo. En este sentido, hay dos niveles en los cuales

10 El desarrollo creativo y la ejecución pueden se realizados por la propia empresa o pueden ser contratados con alguna empresa especializada. Un ejemplo ilustrativo, desde su propia denominación a su modo de funcionamiento, puede ser www.eventosquedejanhuella.com, del grupo Inforpress, cuya metodología -tal y como estamos defendiendo-resulta de principios claros como la creación de acontecimientos sólo desde objetivos concretos de la empresa y desde la necesidad de obtener de ellos resultados medibles, comprobables.

11 Existen un buen número de definiciones sobre Imagen corporativa. Levionnois (1992:204) la entiende como "cualquier percepción consciente o subconsciente provocada por informaciones más o menos racionales, instantáneas o repetitivas, procedentes de un objeto, de una organización, de un hecho o de un acontecimiento"; para Dowling (1994:8) es "la impresión total que una organización genera en la mente de los públicos"; también, para Carrascosa (1992:29) la imagen corporativa es "el resultado neto de la interacción de todas las experiencias, creencias, sentimientos, conocimientos e impresiones que los diferentes públicos internos y externos perciben respecto a una organización". 
debemos trabajar: el de la notoriedad de nuestra imagen (cuánto nos conocen) y el de la calidad de la imagen (cómo nos conocen), un doble análisis que debemos realizar de forma complementaria y siempre que sea posible para cada uno de los públicos que hemos definido como prioritarios ${ }^{12}$.

Dentro del análisis de la imagen, es imprescindible mencionar los estudios de Imagen Publicada, denominación con la que hacemos referencia a la exploración sistemática de la presencia de la compañía en los medios de comunicación. Mencionamos este punto porque existe, todavía, una verdadera obsesión por parte de muchas empresas y directivos en conceder a esta dimensión una importancia que puede considerarse desmesurada, sobre todo cuando hablamos de medios generalistas. Olvidan, estos directivos, que lo relevante no es medir nuestra visibilidad en los medios sino si esa visibilidad tiene algún tipo de consecuencia en nuestros públicos.

Al margen de ello, es indudable que la imagen publicada tiene su parcela de importancia para la empresa, puesto que ofrece pistas útiles sobre los problemas o las carencias que puede presentar su imagen pública. Por ello todas las grandes compañías recogen diariamente lo publicado por los medios de comunicación para elaborar el press-clipping ${ }^{13}$ que remiten a los directivos de la organización. Pero, la parte más interesante llega después, cuando podemos analizar con pausa estas apariciones. Para ello, es habitual codificar todo el material a través de una base de datos diseñada a partir de la metodología propia del análisis de contenido. Con el tiempo, los datos acumulados, muestran las regularidades más importantes que caracterizan a nuestra imagen publicada y, también, las zonas oscuras sobre las que tendremos que orientar la comunicación en el futuro.

12 Algunas organizaciones incluyen también estudios relacionados con la Reputación Corporativa, un tipo de estudios que ha conseguido cierto éxito en nuestro país y que, por lo tanto, ocupa parte de los recursos de investigación de imagen en un buen número de empresas. Un ejemplo muy claro es el banco BBVA, que entiende la reputación como el conjunto de percepciones que tienen sobre la empresa los diversos grupos de interés con los que se relaciona, tanto internos como externos. La reputación -afirma el propio bancoes el resultado del comportamiento desarrollado a lo largo del tiempo y describe la capacidad de la organización para entregar valor a los mencionados grupos. BBVA desarrolló en 2005 un modelo de medición y gestión de la reputación corporativa, modelo que en su caso está estrechamente vinculado a la gestión de la responsabilidad corporativa, siendo gestionado directamente por el departamento de Responsabilidad y Reputación Corporativas.

13 El Press Clipping es, sin duda, uno de los instrumentos más utilizados desde los antiguos gabinetes de prensa. Se trata de la recogida regular y sistemática de los textos publicados por los medios de comunicación que tengan como eje central nuestra compañía y, en muchos casos, también las de nuestro sector. A pesar de los problemas crecientes de carácter legal de estos materiales (las editoras de prensa diaria han demandado a las empresas de Press-Clipping al considerar que se vulnera su derecho a la propiedad intelectual y se les hace "competencia desleal"), lo cierto es que es una práctica muy extendida puesto que este material es muy aprovechable internamente, tanto por el conocimiento directo de los directivos de lo que ocurre en los medios a través de un vehículo manejable y sencillo, como para el comunicador, como mapa sobre el que trazar estrategias de comunicación para la compañía en el futuro. 


\subsection{Estudios de Comunicación}

En no pocas ocasiones, los profesionales denominan a esta segunda línea de trabajo Auditoría de Comunicación, tratando de definir estas labores como las del auditor que analiza y valora el funcionamiento de los principales resortes de comunicación de la compañía, tratando de encontrar sus carencias o vacíos. Se trata, por ello, de conocer y evaluar los procesos de comunicación, los canales e instrumentos, e incluso la temática y contenido de las relaciones entre la organización y sus públicos prioritarios. En esta auditoría, que puede ser de tipo global o bien centrada en aspectos concretos o en problemas ya detectados desde la organización, se evalúan cuestiones como la necesidad de determinadas informaciones por parte de los públicos, los canales que se utilizan y los que no, los mensajes que reciben los públicos, su claridad y credibilidad o hasta la valoración de los productos de comunicación de la compañía. Lo ideal, en todo caso, es realizar este análisis para cada uno de los públicos.

A partir de las preguntas adecuadas, es posible obtener la información significativa sobre la cual efectuaremos los cambios pertinentes en el diseño del sistema de comunicación de la compañía. Dentro del diseño, y como parte esencial de toda política comunicativa, se encuentra la creación de un manual de comunicación, documento en el que se recoge la política de comunicación de la compañía, incluyendo normalmente, la explicación del modo en el que se organiza la comunicación, las normas generales de comunicación y el mapa de públicos. Si bien este manual debe aplicarse también a la dimensión comercial e interna de la comunicación, es indudable el peso que en el conjunto representa la dimensión externa. En todo caso, el manual de comunicación debe ser un documento renovado y actualizado cada año teniendo en cuenta los progresos del último ejercicio, las nuevas necesidades de imagen de la organización o los cambios cualitativos en los públicos.

\section{Comunicación en Internet}

Si hay un ámbito en donde la comunicación corporativa está dando sus primeros pasos serios, éste es el de Internet. Se trata no sólo de crear y gestionar la web corporativa de la compañía -algo que con mayor o menor éxito han acometido buena parte de las empresas-, sino de aprovechar los recursos y posibilidades que proporciona la red de redes en el momento actual, algo que va más allá del mero posicionamiento de la empresa en Internet, para tratar de alcanzar otro tipo de relaciones con los públicos participantes en la que se ha dado en denominar web 2.0. Se trata de sacar un rendimiento neto a los blogs o bitácoras (tanto en los ajenos como los que se puedan crear en la propia compañía, algo cada vez más habitual ${ }^{14}$, en parti-

14 Existen muchos y variados casos, sobre todo en EEUU, que también en esto nos lleva la delantera. Así, Nike creó un blog donde 15 
cipar en comunidades virtuales, en foros o en cualquier otro tipo de iniciativa que permita fomentar un contacto mucho más directo, emocional y profundo con los internautas que, en numerosas ocasiones, funcionan como verdaderos prescriptores de opinión para un buen número de personas y cuya influencia puede incluso llegar a los medios de comunicación convencionales. En esta dimensión, el éxito de la comunicación radica en la relación entre ambas partes, creando un espacio de transparencia, diálogo, reflexión y, por qué no, de crítica, es decir, información útil y de calidad que al comunicador le será muy útil para testar el estado de opinión fuera de la organización.

Las responsabilidades del comunicador en este terreno incluyen además, y lógicamente, otros aspectos más comunes, como los criterios de diseño y actualización de la web corporativa de la organización (de toda ella, no sólo de la parte reservada a la comunicación), el mantenimiento y gestión de la Sala de Prensa a la que hemos hecho referencia en las páginas anteriores, o la articulación y control de la denominada "dark site", web corporativa que la empresa mantiene oculta para hacer pública en el caso de que estalle una crisis que le afecte. En los próximos años, los recursos de Internet ampliarán las posibilidades de la comunicación corporativa, como destaca López Triana (en Villafañe, 2008: 21), cuando habla de las nuevas opciones propiciadas por la tecnología 2.0, asegurando que no sólo conllevará saber qué es un blog, sino que implicará, sobre todo, "entender una nueva cultura de la comunicación corporativa acorde al hilo de los tiempos. La resistencia de los profesionales de la comunicación, de las empresas, no es tanto técnica ante las nuevas herramientas (...), sino quizás también cultural, sobre todo cultural”.

\section{RSC, Patrocinio y Mecenazgo}

Un campo en el que es posible observar un importante crecimiento estos últimos años es el de la "actividad social" de la empresa, tanto a nivel puramente organizacional como a nivel comunicativo. Desde hace muchas décadas -tal y como comentan Andrés (1993), García Perdiguero (2003) o Villafañe (2003)-los gestores de la comunicación han entendido que la comunicación de la vertiente social de la empresa es una importante dimensión para su proyección positiva y que, por lo tanto, es importante su gestión desde un punto de vista comunicativo. Las personas juzgan cada vez más a las compañías en función de sus valores y comportamientos en la sociedad y, en esa línea de percepción social, encajan perfectamente el desarrollo del

prestigiosos artistas propusieron durante 20 días su interpretación de “speed”, concepto asociado a su marca; Amazon habilitó blogs para los autores de los libros que comercializa, desde donde pueden intercambiar comentarios con sus lectores; Barbie, en el site de myscene, habilitó blogs para cada una de sus muñecas, donde a modo de diario, publican sus ficticias pero adolescentes historias o IBM, que difunde los blogs personales de sus programadores, en un ejercicio de participación horizontal que ya se transformó en caso de estudio. 
Patrocinio o el Mecenazgo y, crecientemente, de la Responsabilidad Social Corporativa (RSC o también denominada RSE).

La gestión comunicativa de la RSC ha sido una de las líneas que mayor crecimiento ha experimentado estos últimos años. Los gestores parecen haberse dado cuenta de que cada vez es más necesario desarrollar prácticas responsables en el comportamiento diario de la empresa, un mínimo cada vez más extendido para buena parte de ellas, especialmente las más grandes y las que desarrollan su actividad en sectores socialmente más sensibles. La RSC no deja de ser un compromiso explícito sobre la conducta como organización en relación con sus públicos.

Las grandes compañías han abandonado la mera difusión de mensajes informativos y publicitarios de sus productos o marcas para pasar a presentar sus compromisos como organización social al conjunto de sus públicos. Así, los mensajes cambian, y hasta cambia la organización. Así, Endesa reclama con el mensaje (y la web) "para los hijos de tus hijos" el compromiso y el desafío de una nueva manera de estar y vivir en el planeta, que debe empezar por su propia organización; o Repsol, que asume con su eslogan "inventemos el futuro", su compromiso en desarrollar "soluciones energéticas responsables".

Todo este conjunto de mensajes y manifestaciones concretas a las que obliga la RSC debe estar íntimamente unido a la dirección de comunicación de la compañía y debe ser gestionado con profesionalidad y coherencia sin caer en prácticas que traten sólo de legitimar socialmente la actividad de la empresa o como mera estrategia de maquillaje ético puramente comercial.

\section{Comunicación del Presidente}

Otra de las funciones de comunicación que mayor proyección pueden proporcionar a la compañía -algo indispensable en todas las grandes empresas- es la comunicación propia del presidente ${ }^{15}$. Si analizamos críticamente la realidad, sobre todo en las grandes corporaciones, nos damos cuenta de que la imagen de estas compañías es inseparable de la imagen de sus líderes, presidentes o fundadores ${ }^{16}$ : Isidoro Álvarez para El Corte Inglés, Francisco González para el BBVA, César Alierta para Telefónica o Emilio Botín para el Grupo Santander. Incluso, en casos excepcionales, la "invisibilidad" de su líder ha sido, voluntaria o involuntariamente, una política de imagen perfectamente definida (el caso más visible es el de Amancio Ortega, presidente del Grupo Inditex).

15 Presidente o directivo más importante de la compañía, es una denominación genérica.

16 De hecho, es posible conocer el ranking de los líderes empresariales de mayor prestigio a través del Merco Líderes, el Medidor de Reputación Corporativa elaborado por Villafañe y asociados (www.merco.info/ver/mercolideres/que-es-merco). 
En general, los principios del branding ${ }^{17}$ son directamente aplicables a la gestión de la imagen del líder de la compañía. Se trata de investigar sus posibilidades comunicativas y trazar las líneas básicas del perfil del directivo que hay que posicionar, en este caso, en la mente de los distintos públicos con los que tiene relación, tanto dentro como fuera de la organización. Nada hay más útil para la buena marcha de una organización que la existencia de un líder que, por una parte, sepa motivar y orientar a sus empleados y que, por otra, tenga una presencia pública relevante que sea capaz de generar valor a la organización.

Para lograr este valor personal para el líder de la compañía son imprescindibles tres cosas: una investigación cuidadosa y sistemática sobre las opciones de imagen de nuestro líder; un entrenamiento adecuado y regular sobre las principales herramientas de comunicación que se deben poner en práctica para la consolidación de su imagen; y, por último, el diseño de un conjunto de acciones de comunicación en las cuales su "marca personal" tenga una presencia significativa y adecuada a nuestras expectativas ${ }^{18}$. Por supuesto, la labor del comunicador es también la de vigilar las amenazas de la imagen de su líder y las diferentes orientaciones que puede ir tomando en función de las circunstancias.

\section{Identidad visual}

La identidad visual de la organización, sus normas y signos de identificación, es uno de los modos a través de los cuales la organización se hace visible para muchos de los públicos con los que se relaciona, sobre todo con los públicos externos. No hay que olvidar que la identidad visual no es más que la traducción simbólica de la identidad corporativa de la organización y que, en muchos casos, no sólo representa a la compañía, sino que la sustituye. De ahí su importancia como resorte comunicativo privilegiado y de ahí la necesidad de su gestión por parte del responsable de comunicación externa.

Por ello, debe ser responsabilidad directa del encargado de comunicación, la gestión y, sobre todo, la vigilancia de este capital simbólico de la compañía para que no distorsione la imagen pública que estas representaciones pueden generar en el conjunto de sus públicos. Esto no quiere decir que el responsable de comunicación tenga los conocimientos de un diseñador, sino que -como aclara Xifra (2007: 285)- conozca "los mecanismos de ideación y creación, ya que nadie sabrá mejor que ellos cuál es el objetivo de

17 Denominación anglosajona con la que se hace referencia a la creación y gestión de marcas (aplicadas a realidades físicas -productos-, territoriales -ciudades, países- o personales).

18 Existen un número indeterminado de posibilidades para ello, desde su presencia en los medios de comunicación (de forma directa o indirecta a través de la firma de artículos de opinión), hasta el diseño de discursos, comparencias públicas, reuniones y encuentros, etc. 
comunicación y de relación que se pretende con la transmisión de la identidad". Villafañe (1999: 68) destaca que, entendida así, como parte de las responsabilidades de comunicación, es posible realizar una política más eficaz de la identidad visual "en la medida en que tanto su concepción como las posibles disfunciones que en ésta puedan darse dependerán de factores (...) que la trascienden pero que constituyen el eje de la gestión corporativa globalmente considerada”.

Si bien la identidad visual se extiende a todos los modos de presentación de la organización a sus diferentes públicos, no es menos cierto que existen ciertos elementos simbólicos que con mayor regularidad sirven de presentación o acompañamiento a la empresa, como su logotipo, sus colores y su tipografía corporativa. Al menos, estos elementos deben ser considerados y gestionados estratégicamente como productos directos de comunicación frente a los públicos externos. Al margen de éstos pueden añadirse otros, como los códigos de estilo lingüístico y fonético, los códigos de estilo audiovisual y literario o las constantes espaciales características de la empresa. Incluso algunas compañías, quizás con una orientación más comercial, comienzan a explorar nuevos territorios para lograr la identificación de la compañía en sus públicos. Es el caso de Telefónica, quien ha decidido crear su propio aroma como una parte más de su identidad corporativa con el objetivo de 'atacar' el sentido que más perdura en la mente del consumidor ${ }^{19}$.

La identidad visual no sólo implica la mera creación o aplicación del clásico manual de identidad visual, sino que se debe construir una verdadera "política visual" para la organización, que alcance, cuando sea posible, a los siguientes "soportes": señalética, merchandising, vehículos de la empresa, distinto tipo de equipamiento, arquitectura, productos y servicios, packaging, material gráfico y publicidad. Un grupo amplio de instrumentos para la transmisión visual de la identidad de la organización, un modo muy directo de "comunicarla".

\section{Gestión y control de la Publicidad}

Otra de las responsabilidades complementarias del comunicador externo es sin duda la gestión de la publicidad que, igual que en el caso de la Identidad Visual, no debe ser más que una responsabilidad compartida con los encargados de la comunicación comercial de la compañía, en un intento de que todas las dimensiones de comunicación de la empresa vayan en la misma dirección y no funcionen de forma independiente. Como asegura Alameda (Losada, 2004: 317), "aunque son dimensiones diferentes (...) la gestión actual de la comunicación apuesta por una integración de las tres dimensiones -corporativa, es-

19 “Aquí huele a Telefónica”, en www.expansion.com (consulta, junio 2008) 
tratégica y comercial- bajo una perspectiva común, de tal manera que la empresa sea gestionada como una globalidad y que todas estas formas distintas de comunicación sean coordinadas, integradas y gestionadas corporativamente".

La labor del comunicador externo debería limitarse en este terreno a participar en las decisiones que se adopten en el terreno publicitario para asegurar que son compatibles con la comunicación externa de la compañía y, en general, con la política de comunicación de la organización. En el caso, muy común, de que un único órgano de comunicación centralice todas las decisiones comunicativas -incluso, en la misma persona-, la implicación del comunicador debería ser mayor y participar directamente en todo el proceso de la estrategia publicitaria, en el copy strategy, en la estrategia creativa y en la estrategia de medios. Buena parte de este trabajo, sin embargo, suele ser encargado a una agencia de publicidad externa para que la desarrolle a partir de las indicaciones de la compañía. Del mismo modo, puede trabajarse en cuestiones comerciales complementarias como el marketing directo, la promoción de ventas, la publicidad en el lugar de venta y otras.

En definitiva, las páginas anteriores han tratado de mostrar de modo resumido los diez frentes en los cuales la comunicación corporativa tendrá su recorrido natural en los próximos años, una vez superado el período en el que su existencia era más una reivindicación ambigua que una realidad útil. La existencia de esta decena de ámbitos de trabajo permitirá ordenar mínimamente las responsabilidades profesionales propias de un gestor de comunicación corporativa y, a la vez, situar en el contexto más adecuado los desarrollos teóricos que irán incrementándose exponencialmente en los próximos años. Al margen de ello, la realidad del mundo empresarial es mucho más compleja y heterogénea, y estas pautas genéricas deben ser acopladas adecuadamente a cada compañía para que puedan contribuir verdaderamente al funcionamiento y al desarrollo de la organización y a su proyección externa.

\section{Referencias bibliográficas}

Álvarez, T. y Caballero, M.(1997): Vendedores de Imagen. Barcelona: Paidós.

Andrés, A. J. (1993): Mecenazgo y Patrocinio. Editmex: Madrid.

Bell J.I. (2004): Comunicar para crear valor. Pamplona: Eunsa.

Canel, M.J. (2007): Comunicación de las instituciones públicas. Madrid: Tecnos.

Capriotti, P.(2007): “La Responsabilidad Social Corporativa de las empresas españolas en los Medios de Comunicación”, Zer, no 23. Universidad del País Vasco. 
Capriotti, P. (1999): Planificación estratégica de la imagen corporativa. Barcelona: Ariel.

Carrascosa, J.L.(1992): Comunicacción, una comunicación eficaz para el éxito de los negocios. Madrid: CDN.

Cervera, A.L. (2004): Comunicación Total. Madrid: Esic.

Costa, J. (ed.) (2005): Master Dircom: los profesores tienen la palabra. La Paz (Bolivia): Grupo Design.

De la Rosa, M. (2009): “Comunicación y Petróleo”, en Revista de la Comunicación, nºg.

Dowling, G. (1994): Corporate Reputations. Londres: Kogan Page.

Fernández del Moral, J. (2003): “El periodismo de fuente en el marco de la especialización periodística”, en Losada, A. y Esteve, F.: El Periodismo de Fuente. Salamanca: Publicaciones Universidad Pontificia de Salamanca.

García Perdiguero, T. (2003): "Crítica a los enfoques tradicionales de gestión de la comunicación. La responsabilidad social de la empresa”, en Telos. Cuadernos de comunicación, tecnología y sociedad, № 54, segunda época, pp. 1-4. Revista electrónica, disponible en internet: (http://www.campusred.net/telos).

Hernández, M.A., Macías, A. (2009): Comunicación Corporativa: Las relaciones con los medios de comunicación. Salamanca: Publicaciones Universidad Pontificia de Salamanca.

Lammers, J.C. y B. Barbour, J. (2006) : An Institutional theory of organizational communication, Communication Theory, Volume 16, N.3.

López Lita, R. (2003): Introducción a la comunicación financiera. Castellón: Ed. Universitas.

López - Quesada, M. (2003): ;Estamos en Crisis!. Madrid: Dossat 2000.

Losada, J.C. (2002): Prensa e Imagen Corporativa en la Universidad. Murcia: Publicaciones Universidad Católica San Antonio.

Losada, J.C. (coord): Gestión de la comunicación en las organizaciones: comunicación interna, corporativa y de marketing. Barcelona: Editorial Ariel.

Meijer, M-M y Kleinnikenhuis, J. (2006): "Issue news and corporate reputation: applying the theories of agenda setting and issue ownership in the field of Business Communications", Journal of Communication, Volume 56, N.3

Mars, A.: "Los anuncios de siempre ya no venden", diario El País, 14 de julio de 2008.

Martínez, O. (2007): “La importancia de los medios de comunicación como aliados en la estrategia de un dircom”, en Revista de Comunicación, $\mathrm{n}^{\circ}$ 0. Madrid: Wolters Kluwer España.

Méndiz Noguero, A. (2001): Nuevas formas publicitarias. Málaga: Publicaciones Universidad de Málaga.

Ollé, R. y Riu, D. (2009): En nuevo brand Management. Barcelona: Gestión 2000.

Pizzolante, Italo (2006): El poder de la comunicación estratégica. Caracas: Editorial CEC. 
Reyes, M. (2007): “¿Quién es el portavoz”, en Revista de Comunicación, nº 0. Madrid: Wolters Kluwer España.

Saura, P. (2005): La gestión y la comunicación de crisis en el sector de la alimentación y las bebidas. Madrid: Universidad Pontificia de Comillas.

Sotelo, C. (2001): Introducción a la Comunicación Institucional. Barcelona: Editorial Ariel.

Xifra, J. (2007): Técnicas de las Relaciones Públicas. Barcelona: Editorial UOC.

Westphalen, M. y Piñuel, J.L. (1993): La dirección de Comunicación. Madrid: Prado.

Villafañe, J.: (dtor.) (2008): La comunicación empresarial y la gestión de los intangibles en España y Latinoamérica. Madrid: Pearson Educación.

Villafañe, J. (2003): “La empresa responsable”, en Telos. Cuadernos de comunicación, tecnología y sociedad, No 56, segunda época, pp. 1-2. Revista electrónica, disponible en internet: (http://www.campusred.net/telos).

Villafañe, J. (1999): Gestión Profesional de la Imagen Corporativa. Madrid: Ediciones Pirámide.

VV.AA. (2006): Periodistas, Empresas e Instituciones, claves de una relación necesaria, Madrid: Estudio de Comunicación y Demométrica. 